\title{
Beam Tests of Ionization Chambers for the NuMI Neutrino Beam
}

\author{
Robert M. Zwaska, James Hall, Sacha E. Kopp, Huican Ping, Marek Proga, Albert R. Erwin, Christos Velissaris, \\ Deborah A. Harris, Donna Naples, Jeffrey McDonald, David Northacker, Milind Diwan, and Brett Viren
}

\begin{abstract}
We have conducted tests at the Fermilab Booster of ionization chambers to be used as monitors of the NuMI neutrino beamline. The chambers were exposed to proton fluxes of up to $10^{12}$ particles $/ \mathrm{cm}^{2} / 1.56 \mu \mathrm{s}$. We studied space charge effects which can reduce signal collection from the chambers at large charged particle beam intensities.
\end{abstract}

Index Terms - Ionization chambers, ionizing radiation, multiplication, neutrinos, particle beam measurements, space charge.

\section{INTRODUCTION}

$\mathbf{T}$ HE Neutrinos at the Main Injector (NuMI) beamline at the Fermi National Accelerator Laboratory [1] will generate an intense $\nu_{\mu}$ beam from the decays of mesons produced in the collisions of $120 \mathrm{GeV}$ protons in a graphite target. The mesons are focused by magnetic "horns" into a $675 \mathrm{~m}$ evacuated volume to allow decays to neutrinos. A downstream Aluminum/Steel absorber and bedrock absorb the remnant hadrons and muons in the beam, leaving only neutrinos. The facility is expected deliver beam to neutrino experiments, beginning with the MINOS neutrino oscillation experiment, starting in early 2005.

The meson decays $\pi / K \rightarrow \mu \nu_{\mu}$ produce an energetic muon for every neutrino, allowing monitoring and validation of the neutrino beam focusing to be accomplished by monitoring of the muon flux. As in several previous experiments, the muon flux and remnant hadron flux at the end of the decay volume will be measured by arrays of ionization chambers [2], [3], [4], [5].

The beam monitoring system will measure the intensity and spatial distribution of the hadron beam at the end of the decay tunnel, upstream of the absorber, and of the muon beam after the absorber and at several stations in the bedrock. The monitoring system will consist of $2 \mathrm{~m} \times 2 \mathrm{~m}$ arrays of

Manuscript received December 2, 2002; This work was supported by the U.S. Department of Energy, DE-AC02-76CH3000 and DE-FG03-93ER40757, DE-FG02-05ER40896, and the Fondren Foundation.

R. M. Zwaska, J. Hall, S. E. Kopp, and M. Proga are with the Department of Physics, University of Texas at Austin, Austin, TX 78712 USA (e-mail: zwaska@mail.hep.utexas.edu; kopp@mail.hep.utexas.edu).

A. R. Erwin, H. Ping and C. Velissaris are with the Department of Physics, University of Wisconsin at Madison, Madison, WI 53706 USA.

D. A. Harris is with the Fermi National Accelerator Laboratory, Batavia, IL 60510 USA.

D. Naples, J. McDonald, and D. Northacker are with the Department of Physics, University of Pittsburgh, Pittsburgh, PA 15260 USA.

M. Diwan and B. Viren are with the Brookhaven National Laboratory, Upton, NY 11973 USA.

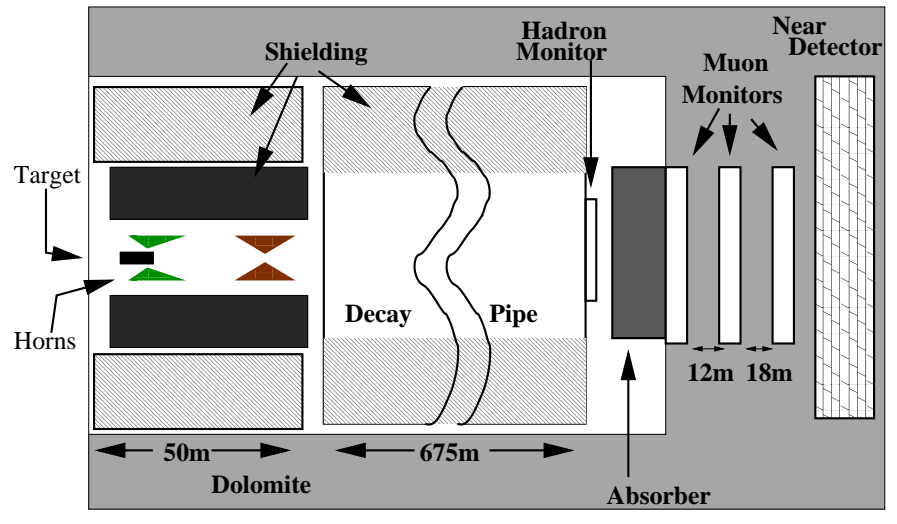

Fig. 1

Pictorial diagram of the NUMI beamline. The 120 GeV PROTON BEAM IS INCIDENT ON THE TARGET PRODUCING A HADRON BEAM. THE POSITIVE HADRONS ARE FOCUSED BY THE HORNS, OF WHICH THE PIONS TRAVEL INTO THE DECAY PIPE WHERE THEY DECAY INTO MUONS AND MUON NEUTRINOS, MAKING THE NEUTRINO BEAM. THE HADRON MONITOR AND MUON MONITORS MEASURE HADRON AND MUON FLUXES AT THEIR LOCATIONS AND ARE CONSTRUCTED OF THE ION CHAMBERS DESCRIBED HEREIN.

ionization chambers with $25 \mathrm{~cm}$ inter-chamber spacing, with one chamber array in each of the above stations. The hadron and muon fluxes are measures of any targeting or focusing failures. The peak charged particle fluxes in one $9 \mu$ s accelerator burst will be $2000,25,3$, and $1.5 \times 10^{6} / \mathrm{cm}^{2}$ in the four monitoring stations.

Each ionization chamber will measure the flux of charged particles by using an applied electric field to collect the ionization created in a helium gas volume. The charge measured from each chamber will be proportional to the charged particle flux at that location. By using an array of chambers the spatial distribution of beam intensity can be inferred. While operated without gas amplification the signal from the intense NuMI beam in one $8 \mathrm{~cm} \times 8 \mathrm{~cm}$ ionization chamber will be 33000 , 1400,170 , and $83 \mathrm{pC}$ at the four stations.

The individual ionization chambers within each array are parallel plate chambers made up of two 4" square ceramic plates with Ag-Pt electrodes. One plate has a single electrode that applies HV bias. The second plate has two electrodes: a central square sense pad measuring 3 " $\times 3$ ", surrounded by a 
$1 \mathrm{~cm}$ guard ring. The sense pad is connected into the electronics which provides a virtual ground. The guard ring is grounded. The chamber gas is pure helium at atmospheric pressure.

The major limitation of ionization chambers used as beam monitors has been space charge build up inside the chamber. Intense particle fluxes release sufficient ionized charge in the chamber gas so as to create a reverse electric field inside the chamber. With the reduced net field in the chamber, ions require a longer time to reach the collection electrodes, and hence suffer more recombination loss in the gas. Recombination losses increase at larger particle fluxes, resulting in a non-linear performance of the ionization chamber beam monitor at large intensities.

\section{Booster BeAm Test}

A beam test of prototype ionization chambers was undertaken at the Fermilab Booster accelerator, which delivers up to $10^{12}$ protons $/ \mathrm{cm}^{2}$ of $8 \mathrm{GeV}$ in a $1.56 \mu \mathrm{sec}$ spill. Tested were two chambers, one with a $1 \mathrm{~mm}$ electrode spacing, the other with $2 \mathrm{~mm}$, with continuos gas flow. We studied the shape of the ionization vs. voltage plateau curve at several intensities and the linearity of the chamber response vs. beam intensity at several applied voltages.

For our beam test we placed two ionization chambers in the beamline. The chambers were housed in a stainless steel vessel with $.005 "$ Ti beam entrance and exit windows. Electrical feedthroughs were made with stainless steel compression fittings and PEEK plastic.

Two gas mixtures were used in the beam test. The primary gas was pure helium supplied from a cylinder with $99.998 \%$ purity. The other gas was a mixture consisting of $98 \%$ Helium and $2 \%$ Hydrogen, with $\leq 20$ p.p.m. of impurities. The gas was first passed through a getter and gas analyzer. Online measurement indicated impurites of $<1.5$ p.p.m. All gas seals were metallic consisting of compression fittings or copper gaskets compressed by vacuum fittings.

Upstream of our ion chamber vessel Fermilab provided a secondary emission monitor (SEM), to locate the beam. The SEM provided targeting information upstream of the chamber, but was not usable in the analysis because the beam diverged after passing through the SEM, and it was only capable of measuring either the horizontal or vertical profile at any given time. Furthermore, the SEM was insensitive below $2 \times 10^{11}$ protons per pulse. During the portion of datataking above $2 \times 10^{11}$ the SEM indicated constant spot size.

A beam toroid was the primary method of measuring the beam intensity delivered to the apparatus. The toroid was supplied with an amplifier and ADC whose least significant bit was $5 \times 10^{9}$ protons.

Rigidly attached on the outside of the ion chamber vessel were two beam profile chambers fashioned out of G-10 circuit board and epoxy. Each chamber had a segmented signal electrode composed of $1 \times 10 \mathrm{~cm}^{2}$ strips. One chamber provided the vertical profile, the other the horizontal profile. The profile chambers were the primary method for determining beam size.

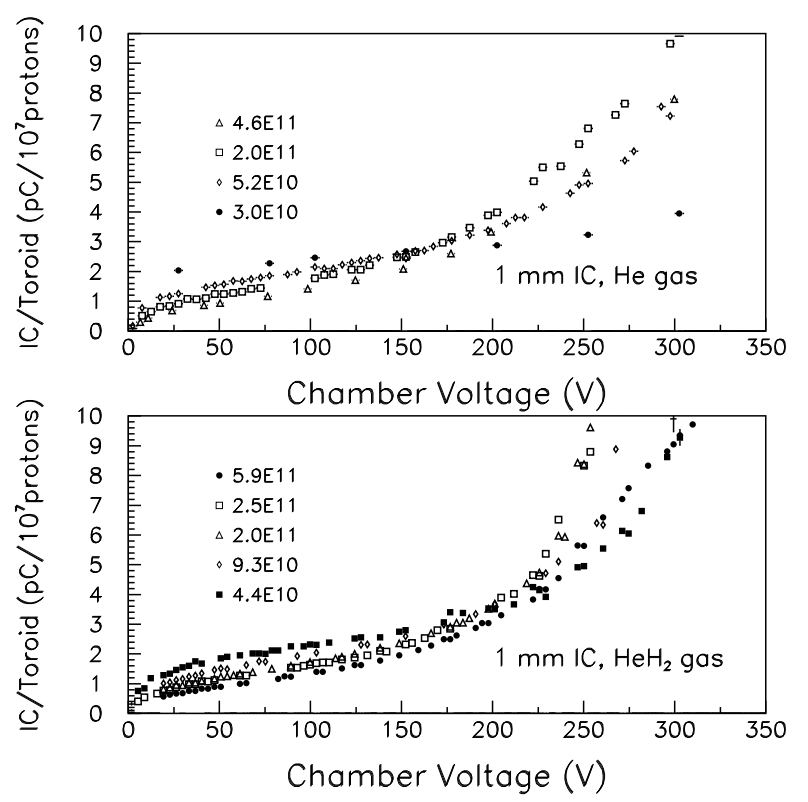

Fig. 2

High VOLTAGE SCANS OF THE 1 MM ION CHAMBER (IC) IN HELIUM AND HELIUM-HYDROGEN AT VARIOUS BEAM INTENSITIES (NOTED IN UNITS OF PROTONS/SPILL). THE VERTICAL AXIS IS THE RATIO OF CHARGE COLLECTED FROM THE IC TO THE BEAM INTENSITY MEASURED BY THE TOROID. EACH POINT IS THE AVERAGE OF 10-20 BEAM SPILLS.

Gas flow was the same as that passed through the vessel. The profile chambers indicated constant beam spot size $\sim 5 \mathrm{~cm}^{2}$.

The signal from each of the ion chambers and beam profile chambers were read out into a charge integrating amplifier and then into an ADC [6]. The electronics were triggered on an accelerator clock signal shortly before the beam pulse. The charge integration time could be altered and taken between beam spills, allowing measurement of pedestals and backgrounds.

\section{EXPERIMENTAL RESULTS}

Tests of the chambers consisted of two complementary measurements. The first held the beam intensity constant, while varying the voltages applied to the chambers. The second held the applied voltages constant while adjusting the beam intensity.

The results of the first test are displayed in Figures 2 and 3 . Each chamber is exposed to several beam intensities and the voltage varied $0-350 \mathrm{~V}$. The ratio of collected charge to the measured beam intensity is plotted as a function of applied voltage.

The ideal voltage plateau curve would consist of a quick rise to a constant charge collected per proton, independent of voltage and intensity. This constant charge collected would be equal to the amount of charge liberated in the gas per proton. At higher voltages gas amplification is expected and charge collected per proton would increase above the plateau. From the height of the plateau on the $1 \mathrm{~mm}$ chamber one may 


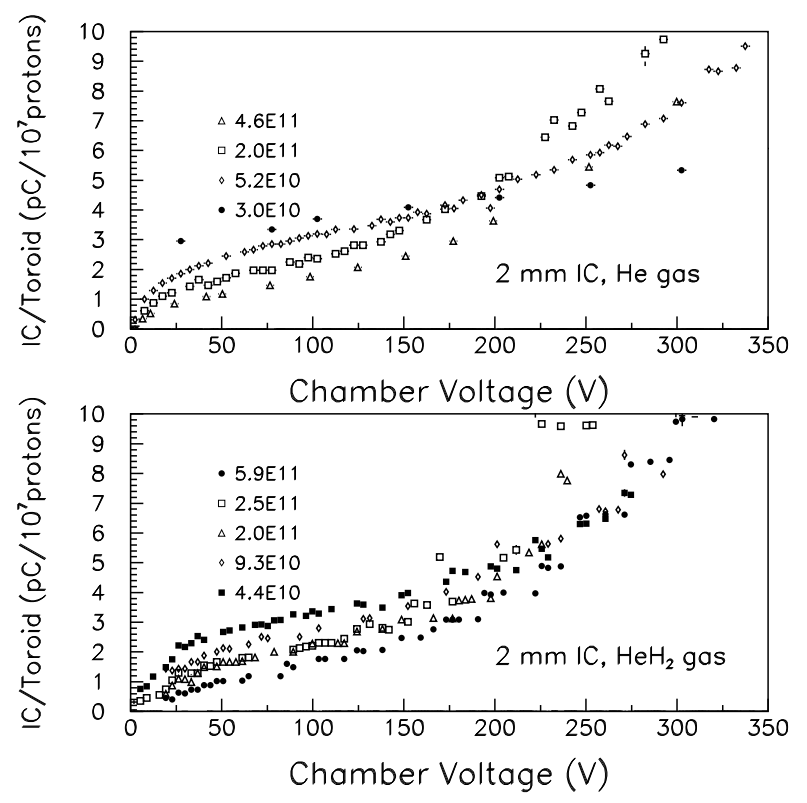

Fig. 3

High VOLTAGE SCANS OF THE 1 MM ION CHAMBER (IC) IN HELIUM AND HELIUM-HYDROGEN AT VARIOUS BEAM INTENSITIES (NOTED IN UNITS OF PROTONS/SPILL). THE VERTICAL AXIS IS THE RATIO OF CHARGE COLLECTED FROM THE IC TO THE BEAM INTENSITY MEASURED BY THE TOROID. EACH POINT IS THE AVERAGE OF 10-20 BEAM SPILLS.

infer a charge ionized per proton of $2.6 \mathrm{pC} / 10^{7}$ protons, or 1.6 electron-ion pairs per proton incident on a $1 \mathrm{~mm}$ gas gap.

The drifting ions and electrons inside the chamber establish their own electric fields and the relatively slow drift velocities of the ions create a net space charge in the gas. This space charge induced electric field screens the electrodes, slowing the transit of ions and electrons across the electrode gap. As discussed extensively in [7], [8], [9], operating ion chambers at very large particle fluences modifies the voltage plateau curve discussed above. If the speed of the charges is sufficiently slowed, recombination may take place. This recombination loss is especially evident $<150 \mathrm{~V}$, where the lower voltages result in slower ion drift velocities and longer ion transit times. This loss also increases at higher beam intensity or in the larger $2 \mathrm{~mm}$ gap chamber, where space charge buildup should be worse.

Space charge effects were evident at all of the intensities delivered by the Booster. In Figure 2 all of the curves, except possibly one, show a slope in the plateau region, suggesting that there is little or no region where the charge is collected without loss or gain. The only useful voltage looks to be $130-190 \mathrm{~V}$ for the $1 \mathrm{~mm}$ chamber, where the curves all intersect. In Section IV we analyze this in terms of competing recombination and multiplication effects in the gas.

Figure 4 displays the results of the second test performed in our beam test for the case of the $1 \mathrm{~mm}$ chamber in Helium. The charge collected from the ion chamber is plotted as a

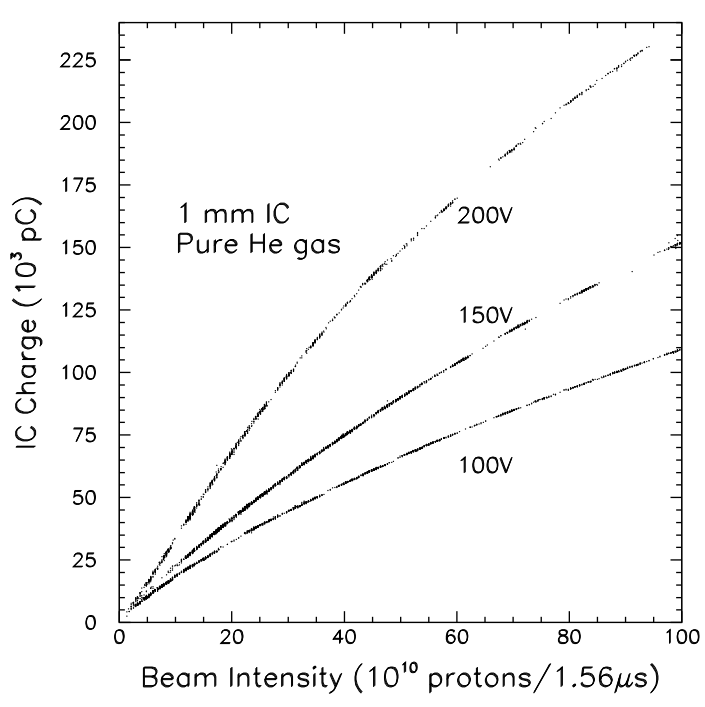

Fig. 4

BEAM INTENSITY SCANS OF THE 1 MM ION CHAMBER IN HELIUM AT VARIOUS VOLTAGES.

function of beam intensity for several applied voltages. Here, ideal curves would all join at low intensity. Recombination loss, especially visible for lower voltages, cause the curves to fall off at higher intensity. The linear region is the operating range for the chamber where charge collected is proportional to incident flux.

An analysis of the $200 \mathrm{~V}$ Helium curve up to $20 \times$ $10^{10}$ protons/spill gives a good linear fit $\left(\chi^{2} / \mathrm{N}_{\text {DOF }}=456 / 625\right)$. However, the intercept is less than zero: $(-0.05 \pm 0.012)$ $\times 10^{3} \mathrm{pC}$. The other data could not be satisfactorily fit to a linear dependence for beam intensities $>10^{10}$ protons/spill.

The nonzero intercept in the $200 \mathrm{~V}$ data is more apparent when the curve is plotted as a ratio, as in Figure 5 where the ratio of charge collected to beam intensity is plotted versus intensity. Here a straight line with zero intercept in the previous plot corresponds to a horizontal line. In Figure 5 the $200 \mathrm{~V}$ curve is obviously curved upward then downward as the intensity is increased from 1 to $20 \times 10^{10}$ protons/spill. This rise is responsible for the negative intercept in the linear fit. In Section IV we discuss how the interplay of multiplication and recombination can cause such an effect. Similar to the voltage plateau data, the data in Figure 5 indicate a collected charge of $\sim 3 \mathrm{pC} / 10^{7}$ protons in the $1 \mathrm{~mm}$ chamber, or 18 electron-ion pairs per $\mathrm{cm}$ in the He.

The similarity between the Helium and Helium-Hydrogen data is interesting. Gas additives with lower excitation potentials are commonly used to increase the drift velocity of electrons [8], which might naively be expected to reduce recombination losses. However, the recombination loss is not substantially changed by the addition of $\mathrm{H}_{2}$, and in Section IV we discuss how the ion drift more strongly affects space charge 

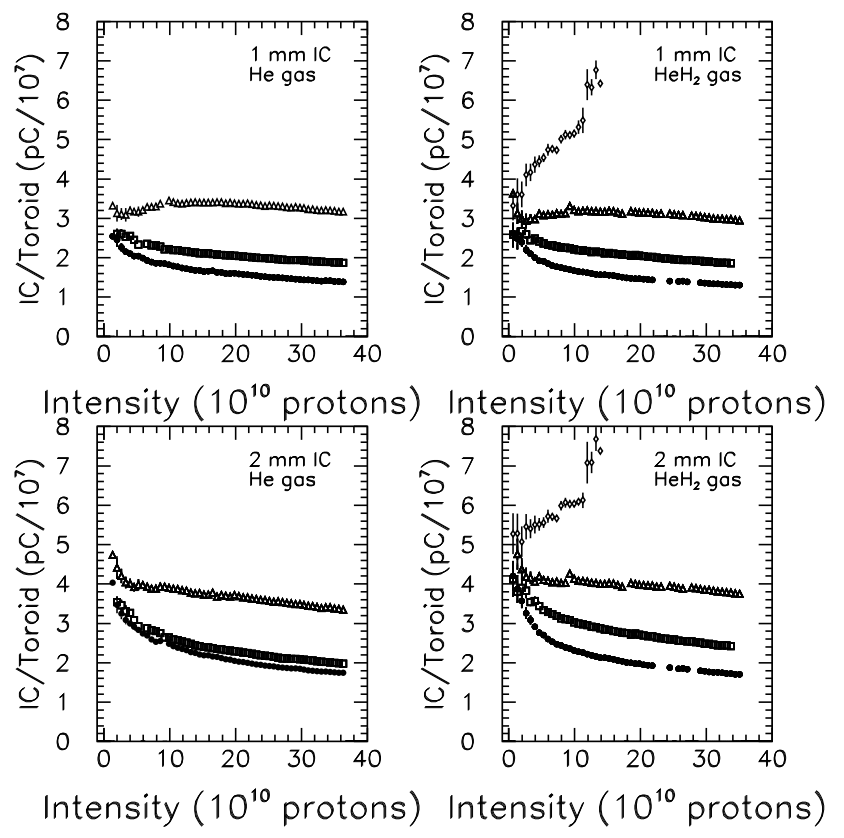

Fig. 5

NORMALIZED BEAM INTENSITY SCANS OF THE 1MM ION CHAMBER AT VARIOUS VOLTAGES. HERE, THE VERTICAL AXIS IS THE RATIO OF CHARGE COLLECTED TO THE BEAM INTENSITY AS MEASURED BY THE TOROID. THE POINTS ARE FOR APPLIED BIAS POTENTIAL OF $100 \mathrm{~V} \bullet, 150 \mathrm{~V}(125 \mathrm{~V}$ FOR $2 \mathrm{MM}-\mathrm{HE}) \square, 200 \mathrm{~V} \triangle$, AND $250 \mathrm{~V} \diamond$.

build-up, hence recombination loss, in the chamber.

Another parameter that might be expected to vary as a result of the additive is the ionization per proton. The incident protons excite metastable states in the Helium, which are followed by collision of Helium atoms with the $\mathrm{H}_{2}$ additive, yielding strong ionization of the $\mathrm{H}_{2}$ [10]. That the observed ionization/proton is not significantly increased by the $\mathrm{H}_{2}$ additive is perhaps indicative that even the minute impurities $(<1.5$ p.p.m. $)$ in the nominally pure Helium gas are sufficient to increase the ionization. The observed 1.6-1.8 ionizations $/ \mathrm{mm}$ per proton in our data is notably higher than the $0.8-1.0$ ionizations $/ \mathrm{mm}$ inferref from the $\mathrm{dE} / \mathrm{dx}$ of fast charged particles and the $w=42 \mathrm{eV} /$ ionization [11] for the purest Helium. Thus, the nominally pure Helium used in typical chamber applications is likely effectively doped.

\section{Simulation}

In this section we model the data collected at the Booster beam test using a computer simulation of the pulse development in an ion chamber. The simulation incorporates the known drift of electrons and of Helium ions in electric fields, the effects of volume recombination of charges, and of gas amplification. The simulation follows the charges during and after the beam spill, recording the net charge collected at the chamber electrodes.

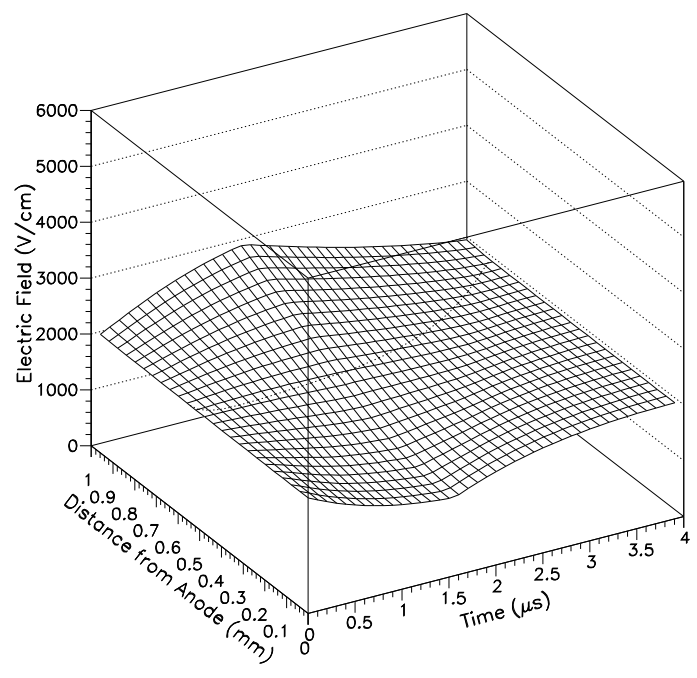

Fig. 6

SIMULATED ELECTRIC FIELD EVOLUTION IN TERMS OF POSITION AND TIME FOR A 1 MM ION CHAMBER OPERATED AT $200 \mathrm{~V}$ WITH IONIZATION OF $10^{10} \mathrm{ION} . / \mathrm{CC} / \mu \mathrm{S}$ FOR $1.6 \mu \mathrm{s}$. SMALL ELECTRIC FIELD DISRUPTIONS DUE TO SPACE CHARGE ACCUMULATION OCCUR AS THE BEAM PULSE DEVELOPS.

The simulations indicate a complex interplay between gas amplification and charge recombination as a result of space charge build up. This calculation will then be used to extrapolate to the particle fluxes and $8.6 \mu$ s spill duration expected in NuMI. Only Helium gas was simulated as literature data for the gas properties of Helium-Hydrogen are minimal, and the beamtest data were not significantly different for the two gases

\section{A. Simulation Method}

The differential equations governing the charge flux and electric field evolution inside the ion chamber are nonlinear. Some earlier work analyzed special cases. 12 demonstrated that space charge accumulation in a continuously-ionized chamber causes the formation of a "dead region" of no electric field, and makes the approximation that all charges in the dead region are lost due to recombination. In [13], an ion chamber ionized over a short duration is studied, but again the assumption is made that all charges in the dead zone are lost to recombination. That assumption is not valid for our experiment where the beam pulse is of short duration allowing the dead zone to disappear before the charge completely recombines.

The present work considers the time dependant case where ionization is delivered in short pulse of $1.6 \mu \mathrm{s}$ and allowed to drift out of the chamber. Our simulation is a finite element calculation of one spatial dimension, such that there are series of infinite planes of charge between the electrodes. The electrons and ions are drifted with the velocites discussed in Section IV-B. Space charge is calculated at each step and 


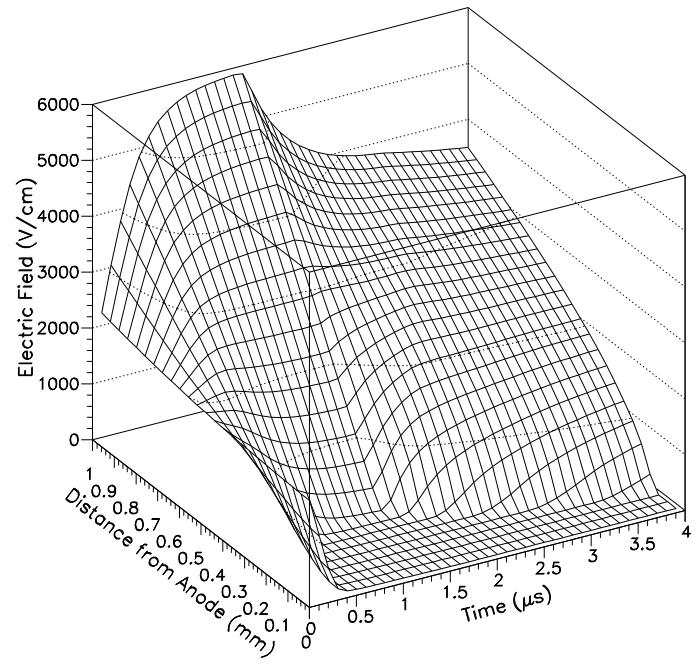

Fig. 7

SIMULATED ELECTRIC FIELD EVOLUTION IN TERMS OF POSITION AND TIME FOR A 1 MM ION CHAMBER OPERATED AT $200 \mathrm{~V}$ WITH IONIZATION OF $10^{11}$ ION./CC/ $\mu$ S FOR $1.6 \mu \mathrm{s}$. A "DEAD ZONE" REGION WITH A VERY LOW ELECTRIC FIELD FORMS AT ABOUT $0.3 \mu$ S INTO THE BEAM SPILL, AND EVENTUALLY GROWS TO COVER A THIRD OF THE CHAMBER. THE SPACE CHARGE ACCUMULATION ALSO INCREASE THE ELECTRIC FIELD IN PART OF THE CHAMBER TO THE POINT WHERE THE MAXIMUM ELECTRIC FIELD AT THE CATHODE IS ALMOST THREE TIMES THE APPLIED ELECTRIC FIELD.

an image charge is induced such that the potential difference betweeen the electrodes is maintained at the applied voltage.

Simulated electric field distributions as a function of time are shown in Figures 6, 7, and 8. The figures show the field development using only the charge transport, and ignore charge recombination or multiplication.

The electric field simulated in Figure 6 is calculated at an intensity of $10^{10}$ ionizations $/ \mathrm{cm}^{3} / \mu \mathrm{s}$, where the excess of ions slightly warps the field. In Figure 7, the ion excess is much greater, to the point where it entirely screens the anode from the applied field, creating a dead zone. As ionization continues in the dead zone the ion and electron densities there increase because the charges are effectively trapped. When the beam spill ends the dead zone slowly fades away. Charge is able to escape from the edge of the dead zone because the dead zone is not created by the charge inside, but the excess of ions outside of it.

Figure 8 is calculated at the same intensity as Figure 7 , but for the $2 \mathrm{~mm}$ gap chamber. The half of the chamber close to the anode has almost exactly the behavior of the $1 \mathrm{~mm}$ case. This similarity is a result of the complete screening of the electric fied by space charge which makes the rest of the chamber in the dead zone irrelevant, until the charge has a chance to excape after the ionization period (the beam spill) ends.

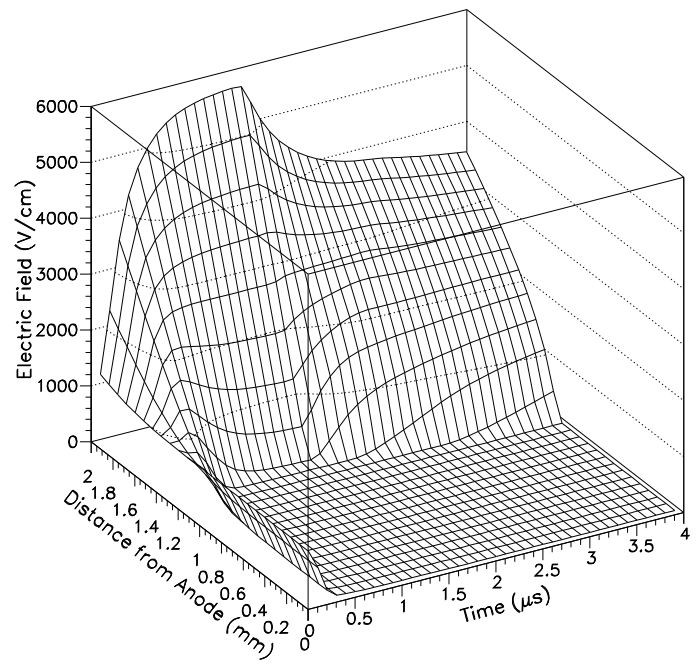

Fig. 8

SIMULATED ELECTRIC FIELD EVOLUTION IN TERMS OF POSITION AND TIME FOR A 2 MM ION CHAMBER OPERATED AT $200 \mathrm{~V}$ WITH IONIZATION OF $10^{11} \mathrm{ION} . / \mathrm{CC} / \mu \mathrm{S}$ FOR $1.6 \mu \mathrm{s}$. A "DEAD ZONE" REGION WITH A VERY LOW ELECTRIC FIELD FORMS AT ABOUT $0.3 \mu$ S INTO THE BEAM SPILL, AND EVENTUALLY GROWS TO COVER MOST OF THE CHAMBER. SHORTLY INTO THE SPILL THE BEHAVIOR OF THE HALF OF THE CHAMBER ADJACENT TO THE CATHODE IS ALMOST IDENTICAL TO THAT OF THE 1 MM CHAMBER IN FIGURE 7

\section{B. Gas Properties}

The parameters of charge drift, loss, and amplification used in this simulation have been taken from several literature sources. The electron drift velocities as a function of electric field are taken from [14]. For the drift of Helium, we use the mobility for the $\mathrm{He}_{2}^{+}$ion, $\mu_{H e_{2}^{+}}=20 \mathrm{~cm}^{2} /(\mathrm{V} \cdot \mathrm{s})$ [14], following the discussion of [10] which notes that above pressures of a few Torr, $\mathrm{He}^{+}$ions tend to collide in the gas and form molecular ions. Previous work [15], [16] have cited $10 \mathrm{~cm}^{2} /(\mathrm{V} \cdot \mathrm{s})$ as the relevant mobility for $\mathrm{He}$.

We simulated charge loss through two- and three-body volume recombination of electrons ions. [14] and [17] parameterize recombination of the form: $d n_{-} / d t=d n_{+} / d t=$ $-r n_{-} n_{+}$, with $n_{+}\left(n_{-}\right)$the positive (negative) ion densities in the gas, and the measured recombination coefficient $r=$ $2.4 \times 10^{-8} \mathrm{~cm}^{3} /($ ion $\cdot \mathrm{s})$.

We did not simulate charge loss through the process of electron attachment to electronegative impurities and subsequent recombination. Using the data from [7] and [17] we find that the attachment of electrons to Oxygen in the gas is insignificant for impurity levels less than 30 p.p.m. (to be compared with 1.5 p.p.m. observed in our chambers). Attachment is negligle over the drift region and occurs primarily in the dead zone where the electrons persist for a greater time. There, the electrons will attach with a time constant $\tau=(67 / x) \mathrm{ms}$, 


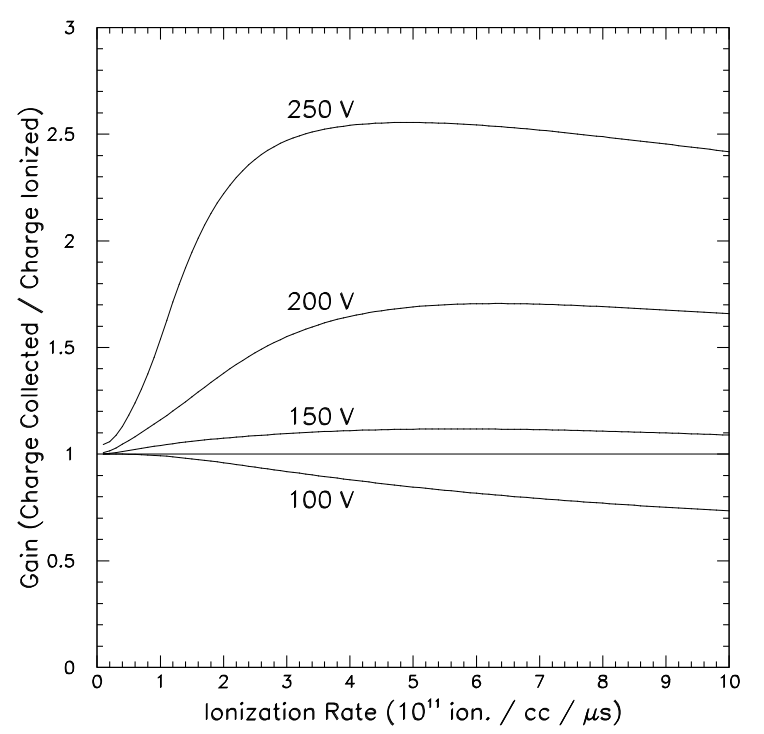

Fig. 9

SIMULATED NORMALIZED INTENSITY SCANS OF A 1 MM IN CHAMBER OPERATED AT VARIOUS VOLTAGES WHERE CHARGE RECOMBINATION AND GAS MULTIPLICATION ARE INCLUDED. THE VERTICAL AXIS IS THE RATIO OF CHARGE COLLECTED TO THE AMOUNT OF CHARGE INITIALLY IONIZED IN THE CHAMBER THE HORIZONTAL LINE AT 1.0 ON THE VERTICAL AXIS IS EXPECTED WHEN THE SPACE CHARGE EFFECTS OF RECOMBINATION AND MULTIPLICATION ARE IGNORED.

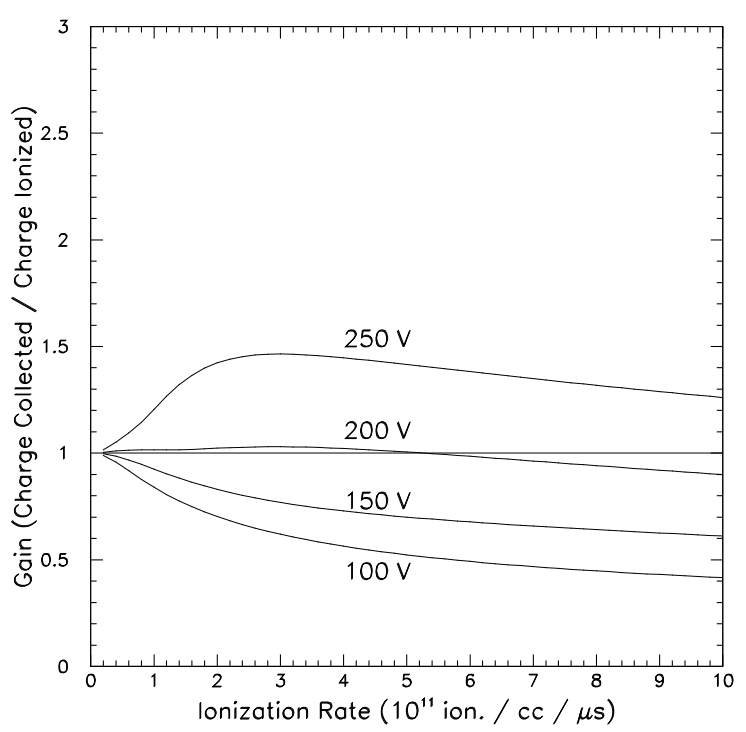

Fig. 10

SIMULATED NORMALIZED INTENSITY SCANS OF A 2 MM IN CHAMBER OPERATED AT VARIOUS VOLTAGES WHERE CHARGE RECOMBINATION AND GAS MULTIPLICATION ARE INCLUDED. THE VERTICAL AXIS IS THE RATIO OF CHARGE COLLECTED TO THE AMOUNT OF CHARGE INITIALLY IONIZED IN THE CHAMBER THE HORIZONTAL LINE AT 1.0 ON THE VERTICAL AXIS IS EXPECTED WHEN THE SPACE CHARGE EFFECTS OF RECOMBINATION AND MULTIPLICATION ARE IGNORED.

\section{Simulation Results}

We found that the simulated space charge accumulation causes not only recombination losses, but the high fields also cause significant multiplication. This result can be seen in Figures 9 and 10 where the charge collected per ionization can actually increase with ionization, until recombination takes over. This should be compared to Figure 5. Both the data and the simulation indicate such an effect. This feature of the curve makes the fit line have a negative intercept, as stated earlier. The lower voltage required in the simulation to generate this effect suggests that multiplication may be overstated in simulation.

The simulation was used to generate voltage plateau curves as well. The results, shown in Figures 11 and 12 , can be compared to Figures 2 and 3, respectively. The behavior is marked by greater losses at high intensity and low voltage, and also by a crossing point where the curves all approach each other. The crossing point occurs when the charge collected equals the charge liberated in the gas. In the simulation this occurs at $\approx 140 \mathrm{~V}$ and $210 \mathrm{~V}$ for the 1 and $2 \mathrm{~mm}$ chambers. In the data, these points occur at $175 \mathrm{~V}$ and and $190 \mathrm{~V}$. Another feature is that higher intensities can show gain at lower voltages, resulting in the curves diverging after the crossing point.

The behavior observed in the data is nominally reproduced by the simulation. The crossing point occurs at a higher voltage than in the simulation of the $1 \mathrm{~mm}$ chamber, again suggesting are used. 


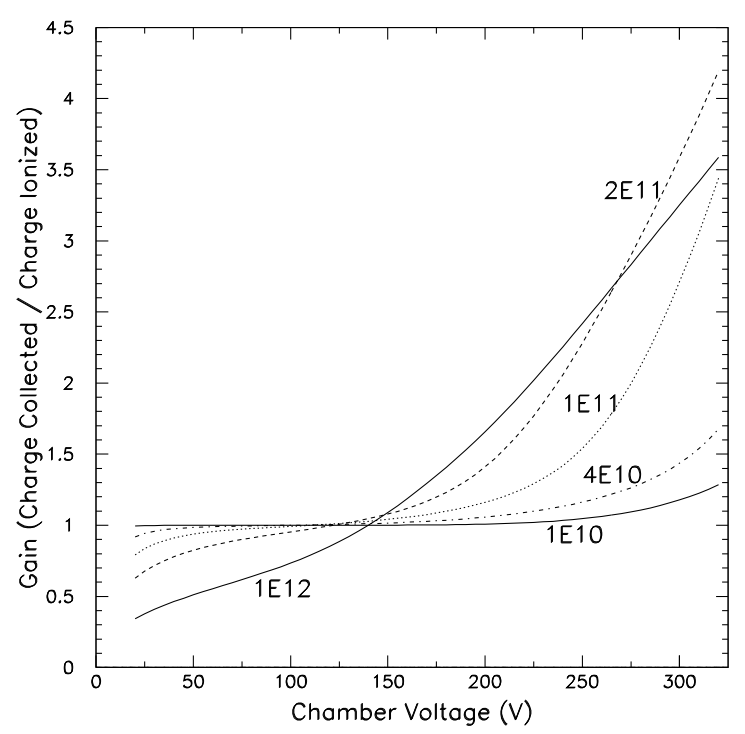

Fig. 11

SIMULATED VOLTAGE SCANS OF A 1 MM ION CHAMBER OPERATED AT VARIOUS INTENSITIES WHERE CHARGE RECOMBINATION AND GAS MULTIPLICATION ARE INCLUDED. THE VERTIAL AXIS IS THE RATIO OF CHARGE COLLECTED TO THE AMOUNT OF CHARGE INITIALLY IONIZED IN THE CHAMBER.

that multiplication is overstated there. The crossing point can be moved left and right by simply changing the value of the Townsend coefficient $\alpha$, and is particularly sensitive to the parameter $B$. Hence the data can constrain the value of $\alpha$, and possibly the recombination coefficient $r$.

\section{CONCLUSION}

We have performed a beam test of Helium- and HeliumHydrogen-filled ionization chambers at the Fermilab Booster accelerator. We have compared the experimental results to our own calculation of the expected charge collection in such chambers. While we have yet to extrapolate our calculations to the anticipated NuMI beam environment using the constraints of our beam test data, several effects of interest are observed. First, calculation of the expected ionization per charged particle in Helium based $d E / d x$ loss and the value $w=42 \mathrm{eV}$ from 11. may ignore $\sim$ p.p.m. impurities in most chamber gas which result in additional ionization. Second, similar to previous work, our results indicate that space charge effects induce recombination losses in the collected charge at high particle fluxes; however, our results indicate additionally that gas amplification occurs in the ion chambers at high beam intensities due to the large space charge build-up of the electric field. The gas amplification gains compete with the losses due to recombination, effectively extending the range of linear response of the ion chamber with respect to beam intensity.

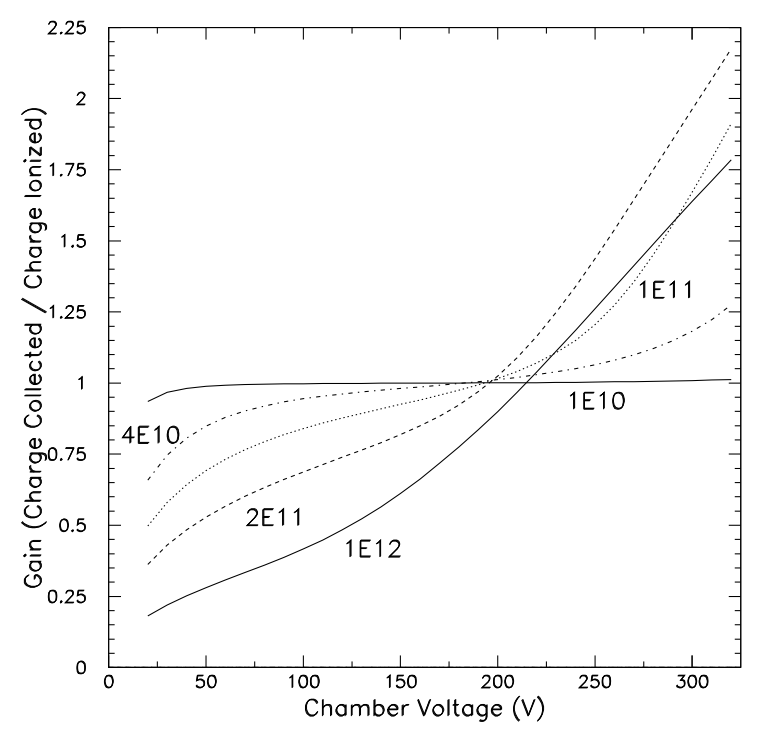

Fig. 12

SiMULATED VOLTAGE SCANS OF A 2 MM ION CHAMBER OPERATED AT VARIOUS INTENSITIES WHERE CHARGE RECOMBINATION AND GAS MULTIPLICATION ARE INCLUDED. THE VERTIAL AXIS IS THE RATIO OF CHARGE COLLECTED TO THE AMOUNT OF CHARGE INITIALLY IONIZED IN THE CHAMBER.

\section{ACKNOWLEDGEMENTS}

We gratefully acknowledge the excellent facilities and technical help provided by the Fermilab Booster Radiation Damage Facility group, J. Lackey, M. Ferguson, T. Sullivan as well as B. Webber, Fermilab Proton Source Department Head.

\section{REFERENCES}

[1] J. Hylen et al. "Conceptual Design for the Technical Components of the Neutrino Beam for the Main Injector (NuMI)," Fermilab-TM-2018, Sept., 1997.

[2] R. Blair et al., Nucl. Instr. Meth. A226, 281 (1984).

[3] A. A. Belkov et al., "Calibration of Muon Detectors for IHEP Neutrino Beam Monitoring System," Serpukhov IHEP preprint IFVE-82-99, Apr. 1982, Proceedings of the Eigth International Symposium on Nuclear Electronics, Dubna, 1975, pp 392-395.

[4] J. E. Hill et al., "Tests of Beam Monitoring Equipment for a Neutrino Experiment," Beam Sci. Technol. 3 18-22 (1998); Int. J. Mod. Phys. A16S1B, 758-760 (2001).

[5] H. Wachsmuth, "Neutrino Beam Monitoring," Proceedings of the Symposium on Beam Intensity Measurement, Daresbury, England, DNPL/RI (1968), pp.68-74; R. Bertolotto et al., Proc. 6th Intl. Conf. High Energy Accel., C.E.A., 11-15 Sept. 1967 (CEAL-2000-1967).

[6] W. Kissel, B. Lublinsky and A. Frank, "New SWIC Scanner/Controller System," presented at the 1995 International Conference on Accelerator and Large Experimental Physics Control Systems, 1996.

[7] D. H. Wilkinson, Ionization Chambers and Counters, Cambridge University Press, Cambridge, 1950.

[8] B.Rossi and H.Staub, Ionization Counters and Detectors, McGraw-Hill, New York, 1949.

[9] G. F. Knoll, Radiation Detection and Measurement, Third Edition, John Wiley \& Sons, Inc., New York, 2000.

[10] L. B. Loeb, Basic Processes of Gaseous Electronics, University of California Press, Berkeley, California 1955. 
[11] ICRU, International Commission on Radiation Units and Measurements, Average Energy Required to Produce an Ion Pair, ICRU Report 31, ICRU, Wahington D. C., 1979.

[12] J. W. Boag and T. Wilson, "The saturation curve at high ionization intensity," Brit. J. Appl. Phys., vol. 3, pp. 222-229, 1952.

[13] S. Palestini, G. D. Barr, C. Biino, P. O. Calafiura, A. Ceccucci, C. Cerri et al., "Space Charge in ionization detectors and the NA48 electromagnetic calorimeter," Nucl. Inst. Meth. A, vol. 421, pp. 75-89, 1999.

[14] J. Dutton, "A Survey of Electron Swarm Data," J. Phys. Chem. Ref. Data, vol. 4 , pp. $577-856,1975$.

[15] F. Sauli, "Principles of Operation of Multiwire Proportional and Drift Chambers," CERN preprint CERN-77-09 (3 May 1977).

[16] W. Blum, L. Rolandi, Particle Detection with Drift Chambers, Berlin, Springer-Verlag, 1994.

[17] S. Brown, Basic Data of Plasma Physics: The Fundamental Data on Electrical Discharges in Gases, AIP Press, New York, 1994.

[18] A. von Engel, Ionized Gases, Springer-Verlag, New York, 1995.

[19] K. Hagiwara et al., "Review of Particle Properties," Phys. Rev. D, vol. 66, 010001, 2002. 